
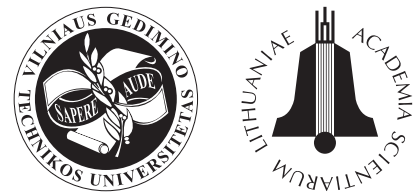

TRANSPORT

2010

25(1): 148-154

\title{
PREDICTABLE UNCERTAINTY ABOUT TERMINAL OPERATIONS IN THE SEA
}

\author{
Svjetlana Hess ${ }^{1}$, Mirano Hess ${ }^{2}$ \\ Dept of Maritime Transportation, Faculty of Maritime Studies, University of Rijeka, \\ Studentska 2, Rijeka, Republic of Croatia \\ E-mails: ${ }^{1}$ shess@pfri.hr; ${ }^{2}$ hess@pfri.hr
}

Received 23 April 2009; accepted 20 May 2010

\begin{abstract}
This paper considers a problem of planning short term operations in a bulk terminal faced by port management when making tactical decisions. Ship loading and discharging, cargo stocking, the maintenance and service of facility equipment are regular operations of a bulk terminal which is a subsystem of a sea port the conduct of which is subject to difficult-to-predict or unforeseen influences. The problem that port management encounters in day to day operations looks into making the best possible plan with the scheduled duration of operations/states and transition instants, considering various internal and external factors influencing terminal performance. A state and transition model is used for deriving effective solutions to obtaining the state order and state transition time of a bulk terminal with an objective of minimizing operational costs. The behaviour of the terminal is tested applying the stochastic and deterministic method.
\end{abstract}

Keywords: operation planning, tactical decision making, sea terminal, theory of general systems, stochastic modelling.

\section{Introduction}

Cargo transhipment in sea ports consisting of numerous operations together with various influences results in specific port behaviour and cargo turnover. In this context, port behaviour means the manner in which port operations take place. In such an operational environment, decisions must be made not only concerning ship scheduling and cargo distribution but also on how to employ port facilities to full capacity, when and how to upgrade facilities, a schedule of repair and maintenance works and plan work shifts. Such decisions are often influenced by the events that cannot be predicted with certainty. The major objective of planning port operations is to diminish port vacancy, thus minimizing operational costs while assuring that service rendered to ships is in line with widely accepted standards (Česnauskis 2007; Hsu and Hsieh 2007; Jaržemskis and Vasilis Vasiliauskas 2007; Afandizadeh and Moayedfar 2008; Paulauskas and Bentzen 2008; Vasilis Vasiliauskas and Barysienè 2008; Imai et al. 2009; Liu et al. 2009; Su and Wang 2009; Chen and Zeng 2010).

During the last decade, a significant amount of attention has been directed towards port/terminal management problems. Veeke and Ottjes (1999) describe the way of detailed modelling and simulation of a new container-handling concept (Improved Port/Ship Interface) of a container terminal that has a direct influence on planning port operations. Cullinane et al. (2005) applied the mathematical programming approach to estimate the efficiency of container port production. Cullinane (2002) also investigated possible methods and their applications for productivity and efficiency modelling ports and terminals.

Even though a wide range of planning problems within shipping industry has received significant attention from researchers so far, there are still problems that have to be addressed, i.e. uncertainty about planning port operations. When dealing with port and ship operations, there is a lot of uncertainty due to weather conditions, mechanical problems and strikes. Thus, optimization under uncertainty is an important field within Operation Research, see the survey by Gendreau et al. (1996). The problem of optimization under uncertainty also exists in bulk terminals, but is of a somewhat different character. Limited storage capacity and facility output necessitate planning terminal operations to prevent storage overflow and unoccupied terminal capacities. In contrast to the vast body of literature dedicated to transportation planning problems, relatively little attention has been directed to the problem of planning port operations. Radić and Bošnjak (1997) formulate the concept of 
the generalized traffic model using the methodology of a general system theory. The model focuses on the level that is not technically specific and describes general traffic behaviour with applicability to different transportation subsystems.

This paper also concentrates on understanding terminal behaviour and addresses the question whether terminal operations show deterministic or stochastic behaviour. The major contribution of this work is made to determining the model of states and transitions (ST model) for bulk terminal behaviour observation which is based on a comparison of deterministic states and transitions (DST method) and stochastic states and transitions (SST method). Besides, we developed a method for a stochastic interpretation of terminal behaviour. The ST model can assist port management in making short term tactical operational decisions, such as planning human resources (shifts planning), maintenance and repair work, facility/machinery engagement in daily/weekly cargo operations, etc. We analyzed the worksheets of a bulk terminal at Bakar port for the period of two years (2007-2008). To define various operations of the terminal for the model, we took data for the year 2007 only. Upon obtaining model results, a comparison considering data on terminal operations in 2008 and conclusions on which the method better fits a real example has been made as well as a proposal for the measures minimizing operational costs has been put forward.

The next section provides a description of the problem and is followed by the mathematical model used to solve it presenting the results of the experiment on the problem of bulk terminal operations. Finally, practical extensions are outlined.

\section{The Problem}

Regular operations of a bulk terminal are ship loading and discharging, cargo stocking, the maintenance and service of facility equipment and distribution of cargo to/from hinterland. One of the major problems and endeavour of port management is to create a plan of the most efficient operations in order to achieve optimal cargo turnover in a manner that will minimize total operating costs and maximize facility capacities. Although the operations are coordinated similarly to those carried out in all real systems (Hess et al. 2007), their conduct is subject to difficult-to-predict or unforeseen influences that may be of internal (machinery breakdown, strike of longshoremen, etc.) or external nature (bad weather, port-hinterland transportation bottleneck, etc.).

The approach taken here in contribution to solving the problem is to consider terminal operations either they are planned or influenced by impact factors as states in which the terminal can be in a given instant. We also take that transitions between states are either planned or subjected to impact factors. We assume that impact factors are stochastic variables since they cannot be predicted with certainty.

The objective of the paper is to identify a particular state in which the bulk terminal will be at a given mo- ment in the future starting from an assumption that in the beginning, it was in idle state and that state switching occurred with designated transition probabilities. Besides, we will try to answer the question whether the observed bulk terminal behaves as a deterministic system, i.e. according to the logical terminal operation flow, or as a stochastic system, meaning that influences causing state transition disorder are not negligible. In the first case, the duration of each state and time of transits at a given period are known with certainty, so there is no need for advanced mathematical procedures to estimate terminal behaviour (Hess et al. 2008). However, in the second case, the impact of odd factors is considerable, and thus the use of an appropriate probabilistic method in order to follow terminal behaviour appears to be necessary.

We set up the ST model of the terminal taking into account its activity, behaviour, states and transitions. A lack of uniformity in the case of cargo arrival at the terminal and the impossibility of predicting the exact time and quantity of cargo arriving on the terminal are the main reasons for the stochastic property of its operations. Since transitions in the DST method are exactly known, at the second stage, we take the effort of quantifying state transitions with the probability distribution of the SST method only. We examine two approaches to quantifying state transitions. The first one consists of setting up a system of differential equations for terminal operations with an assumption that the terminal has discrete states expressed with probabilities. The second approach defining bulk terminal operations as Markov processes and setting up the matrix of transition probabilities yield state probabilities and lead quickly to an accepTable solution to the ST model essential for any practical application.

\section{The Model}

In this section, we set up the ST model of terminal operations. First, we define the DST method for observing deterministic terminal behaviour and develop the SST method for stochastic behaviour.

To simplify a procedure of defining terminal behaviour, we take into account that the terminal exists in one of five states at a given instant. These states include:

- $S_{1}$ - idle state (no operations on the terminal except data processing, i. e. the collection and analysis of weather reports, cargo/ships related information);

- $S_{2}$ - preparatory state (operations carried on the terminal just before ship arrival, i. e. the preparation of facility/cargo/longshoremen for cargo operations);

- $S_{3}$ - transhipment state (cargo loading and/or discharging; from economical perspective, the most desirable state of the terminal);

- $S_{4}$ - closing state (operations performed immediately after finishing ship loading/discharging, i. e. paper-work, ship departure operation);

- $S_{5}$ - repair and maintenance state (regular main- 
tenance of equipment, repair in case of machinery breakdown).

A terminal has deterministic behaviour if the order of states and their durations are exactly known in advance. Having defined only ST-structure with operation flow, one can easily deduce in which state the terminal will be in the future instant. In the DST method, a set of states and transitions between these states for a bulk terminal is formed around ST-structure shown in Fig. 1.

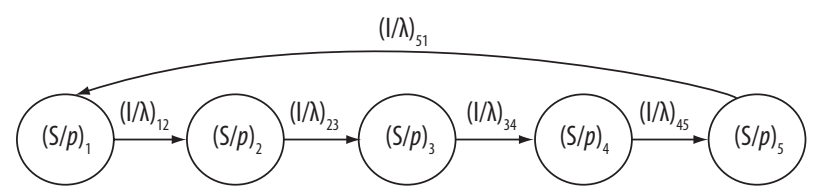

Fig. 1. ST-structure of deterministic terminal behaviour

Transitions between terminal states defined through the DST method are:

- $I_{12}$ - from the idle to preparatory state at ship arrival;

- $I_{23}$ - after ending preparation, transition to cargo transhipment state;

- $I_{34}$ - back to the closing state after transhipment ends;

- $I_{45}$ - from the closing state to maintenance;

- $I_{51}$ - the idle state follows maintenance state.

On a terminal that behaves in a deterministic manner, transitions from state to state follow logical terminal workflow. The occurrence of these transitions is certain. Therefore, in an instant, the transition between adjacent states has probability that equals one while other transitions are not possible.

A terminal has stochastic behaviour if the order of states and transitions do not follow logical workflow due to various internal and external unforeseen influences on regular operations. For researching such a system, the SST method will be developed. In this case, ST-structure may be defined from Fig 2 .

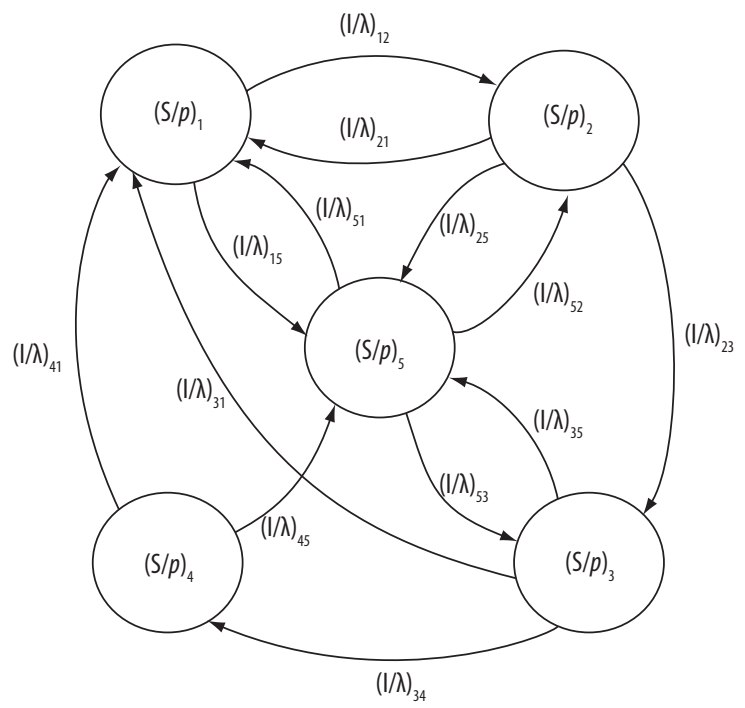

Fig. 2. ST-structure of stochastic terminal behaviour
The SST method, in addition to transitions defined through the DST method, comprises the following transitions:

- $I_{15}$ - from the idle to repair and maintenance state;

- $I_{21}$ - transition from the preparatory to idle state due to bad weather or the strike of longshoremen, etc.;

$-I_{25}$ - breakdown of facility causes transition to repair state;

- $I_{31}$ - back to the idle state if adverse events occur during transhipment;

- $I_{35}$ - breakdown of facility causes transition to repair state;

- $I_{41}$ - to the idle state upon a ship leaves the terminal;

- $I_{52}$ - from maintenance state to the preparatory state due to early ship arrival;

- $I_{53}$ - switch back to transhipment state after failure is removed.

The stochastic flow of operations yields additional transitions between nonadjacent states with various transition probabilities. Since states and transitions are subject to stochastic changes and therefore can be expressed with probabilities that should be quantified for a real example, we set a system of differential equations for the bulk terminal. We derive a system of Kolmogorov equations using the graph of terminal states (Fig. 2):

$$
\begin{aligned}
\frac{d p_{1}}{d t}= & \lambda_{21} p_{2}+\lambda_{31} p_{3}+\lambda_{41} p_{4}+\lambda_{51} p_{5}-\left(\lambda_{12}+\lambda_{15}\right) p_{1} \\
\frac{d p_{2}}{d t}= & \lambda_{12} p_{1}+\lambda_{52} p_{5}-\left(\lambda_{21}+\lambda_{23}+\lambda_{25}\right) p_{2}, \\
\frac{d p_{3}}{d t}= & \lambda_{23} p_{2}+\lambda_{53} p_{5}-\left(\lambda_{31}+\lambda_{34}+\lambda_{35}\right) p_{3}, \\
\frac{d p_{4}}{d t}= & \lambda_{34} p_{3}-\left(\lambda_{41}+\lambda_{45}\right) p_{4} \\
\frac{d p_{5}}{d t}= & \lambda_{15} p_{1}+\lambda_{25} p_{2}+\lambda_{35} p_{3}+\lambda_{45} p_{4}- \\
& \left(\lambda_{51}+\lambda_{52}+\lambda_{53}\right) p_{5},
\end{aligned}
$$

where: $p_{i}$ is the probability of state $i, i=1, \ldots, 5 ; \lambda_{i j}$ is transition probability from state $i$ to state $j ; i, j=1, \ldots, 5$; and $t$ is time.

Since condition $\sum_{i=1}^{N} p_{i}(t)=1$ is satisfied for any $t$, each probability can be expressed in terms of other probabilities and thus diminish the number of equations by one. To solve the system of differential equations for probabilities of states $p_{1}(t), p_{2}(t), \ldots, p_{N}(t)$, the initial probability distribution $p_{1}(0), p_{2}(0), \ldots, p_{i}(0), \ldots, p_{N}(0)$ the sum of which is equal to unity has to be specified. If in a special case the state of terminal $\mathrm{S}$ at the initial moment $t=0$ is exactly known, $\mathrm{S}(0)=s_{i}$, then $p_{i}(0)=1$ and other initial probabilities are zeros. In our case, if the system of differential equations is set on the basis of ST structure, then a solution to the system presents probabilities of finding the terminal in one of the five possible states depending 
on independent variable $t$ which represents time. State equations can be solved by transformation to their Laplace counterparts. The resulting Laplace transform domain equations inverted to obtain their time-domain solutions. To derive and solve a system of state equations based on the user-specified state diagram, computer support is often required. However, a system of differential equations expresses probabilities for each state as the functions of time and can be transformed into a matrix for solution representing the Markov model of linear differential equations for state probabilities. Assuming that ship arrivals at the bulk terminal is Poisson distributed stochastic variable, the operations of the terminal can be presented with a homogenous Markov chain having the following matrix of transition probabilities:

$$
P=\left[\begin{array}{lllll}
\lambda_{11} & \lambda_{12} & \lambda_{13} & \lambda_{14} & \lambda_{15} \\
\lambda_{21} & \lambda_{22} & \lambda_{23} & \lambda_{24} & \lambda_{25} \\
\lambda_{31} & \lambda_{32} & \lambda_{33} & \lambda_{34} & \lambda_{35} \\
\lambda_{41} & \lambda_{42} & \lambda_{43} & \lambda_{44} & \lambda_{45} \\
\lambda_{51} & \lambda_{52} & \lambda_{53} & \lambda_{54} & \lambda_{55}
\end{array}\right],
$$

where: transition probabilities $\lambda_{i j}$ represent transitions from state $i$ to state $j$ between two consecutive state changes. In the matrix, each probability should be quantified separately for the DST and SST method. The initial state is given by a vector of states $P_{0}$ of the bulk cargo terminal: $P_{0}=\left[p_{1}(0), p_{2}(0), \ldots, p_{5}(0)\right]$, where $p_{i}(0)$, $i=1, \ldots, 5$ are the probabilities of states at the initial moment of terminal observation. If the initial state vector $P_{0}$ and the matrix of transition probabilities $P$ are given, then the probabilities of all states of terminal $P^{(n)}$ can be found from the formula: $P^{(n)}=P_{0} P^{n}$ where $n$ denotes the ordinal number of steps, $n \geq 1$.

\section{The Experiment}

\subsection{DST Terminal Operations}

On the basis of ST structure for deterministic terminal behaviour (Fig. 1) and bearing in mind that the transition from the state to the consecutive state is certain, the matrix of transition probabilities for the DST method is given by:

$$
P=\left[\begin{array}{lllll}
0 & 1 & 0 & 0 & 0 \\
0 & 0 & 1 & 0 & 0 \\
0 & 0 & 0 & 1 & 0 \\
0 & 0 & 0 & 0 & 1 \\
1 & 0 & 0 & 0 & 0
\end{array}\right] .
$$

Considering the simplicity of the matrix, the probability of states after $n$ steps can be easily obtained. For example, following 12 steps, the terminal will be in $S_{3}$ (transhipment state) with probability equal to one. Limiting distribution for Markov process $\left\{X_{n}\right\}$ is defined by:

$$
\omega_{j}=\lim _{n \rightarrow \infty} p_{i j}^{(n)}=\lim _{n \rightarrow \infty} \mathrm{P}\left\{X_{n}=j \mid X_{0}=i\right\} .
$$

Additionally, transition probability matrix $P=\left[p_{i j}\right]$ has the property of a doubly stochastic matrix since:

$$
p_{i j} \geq 0 \text { and } \sum_{k} p_{i k}=\sum_{k} p_{k j}=1 \text { for all } i, j .
$$

Given that the matrix is regular, then unique limiting distribution is uniform distribution $\omega=(1 / 5, \ldots, 1 / 5), i=5$. There is only one solution to $\omega_{j}=\sum_{k} \omega_{k} p_{k j}$ and $\sum_{k} \omega_{k}=1$ when $P$ is regular, so we need only to verify that $\omega=(1 / N, \ldots, 1 / N)$ is a solution where $P$ is doubly stochastic in order to establish the claim. From doubly stochastic property $\sum_{j} p_{j k}=1$ follows: $\frac{1}{N}=\sum_{j} \frac{1}{N} p_{j k}=\frac{1}{N}$.

Therefore, limiting probability in the long run $(n \rightarrow \infty)$ of finding the Markov chain in state $j$ is approximately $1 / 5$, no matter in which state the chain began at time 0 . Furthermore, in our case, $\omega_{j}$ gives the long run mean fraction of time that terminal is in state $j$.

\subsection{SST Terminal Operations}

Data derived from terminal work were used to assemble a problem of stochastic terminal operations. To define various operations in the terminal applying the SST method, we analyzed the worksheets of the bulk terminal in Bakar port for the year 2007. The performed operations include the transportation of bulk cargo from/to terminal, loading/discharging cargo to/from ships, the inspection of ship and cargo, the distribution of cargo to shore stock, the maintenance and repair of facility equipment and customs procedures. We also took data on the frequency of machinery failure, bad weather and strike caused stoppages of operations and congestions on the terminal. These data served as a basis for the population of the stochastic matrix of transition probabilities for bulk terminal behaviour (see matrix (6)). Worksheet data on the terminal show that upon the receipt of ship arrival notice the terminal switches from the idle to preparatory state in $98 \%$ cases resulting in the probability of 0.98 . Further probabilities are obtained analogous in respect to their own meaning.

We can summarize the transition probabilities matrix for the states of the bulk terminal in the port of Bakar bearing in mind that the sum of probabilities by rows is one:

$$
P=\left[\begin{array}{ccccc}
0 & 0.98 & 0 & 0 & 0.02 \\
0.02 & 0 & 0.97 & 0 & 0.01 \\
0.02 & 0 & 0 & 0.95 & 0.03 \\
0.37 & 0 & 0 & 0 & 0.63 \\
0.69 & 0.12 & 0.19 & 0 & 0
\end{array}\right] .
$$

The solution was obtained employing computer-assisted evaluation program WinQSB (Chang 2003) having an integrated Markov modelling and simulation tool based on discrete space and continuous-time Markov model. Similarity in the procedure of determining port 
capacity having a different approach can be drawn to Kia et al. (2002) and Wang et al. (2002). After data entry in the transition table, the defined number of periods ( $n=1, \ldots, 12$ steps), the initial state vector of the terminal at time $t=0, P_{0}=[1,0,0,0,0]$ and the probabilities of the five terminal states are obtained and presented in Fig. 3.

Starting from the idle state, simulation shows in which state the terminal will appear most probably after each transition (step).

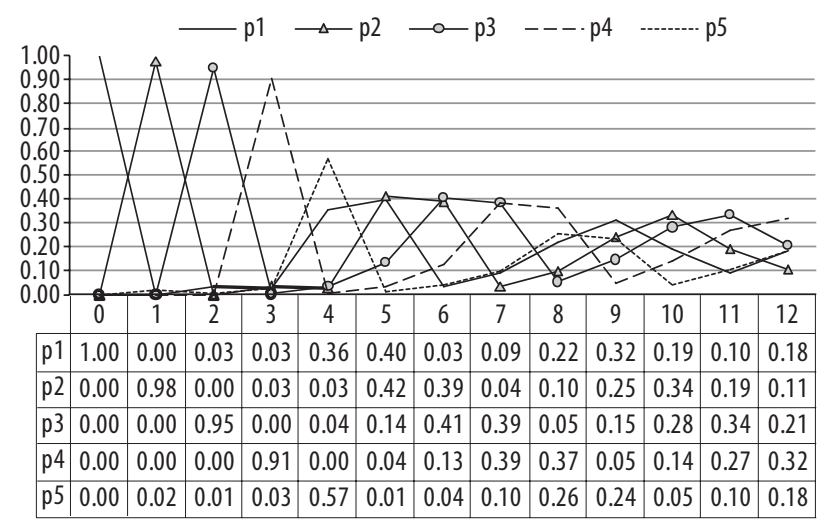

Fig. 3. Probability distribution for terminal states including 12 steps

The probability of the most noTable state decreases with the number of simulation steps and the terminal approaches steady state probabilities. As an illustration, Fig. 3 shows that transitions from state to state do not have to follow a logical order of state transitions (terminal operations).

For example, in the fourth step, the terminal most probably resides in state $S_{5}$ representing the logical position order of the terminal in that step. However, in the next step, the terminal is found with the highest probability to reside in state $S_{2}$ and not in state $S_{1}$ as expected. The reason of such turn in terminal state order can be explained referring to the previously mentioned numerous probabilistic influences impacting terminal operation flow. In order to calculate the average duration of each terminal state, we analyzed terminal work sheets for the year 2007 separately for time intervals associated with ship arrivals when terminal operations had DST behaviour and those with SST behaviour. As predicted, the averages of the duration of states $S_{2}, S_{4}$ and $S_{5}$ have low standard deviation, so we took them as representative values. For states $S_{1}$ and $S_{3}$, deviation from average is high and cannot be taken into further calculations. The duration of state $S_{1}$ depends on the actual time of ship arrival while the duration of state $S_{3}$ is influenced by the size of ships arriving and the quantity of cargo manipulated. The duration of each state in SST terminal operations is generally longer than the equivalent one in DST since, unexpected influences cause additional waiting time and longer working procedures. For illustration, Table 1 shows a seven days period of terminal operation. Besides, we made analogous simulations for 52 weeks taking the same matrix of transition probabilities but starting each week with a different matrix of the initial states formed, considering the real state in which the terminal is found.

Assuming that the terminal switches states from $S_{1}$ to $S_{5}$ in deterministic order, terminal operation flow as well as state duration and the moments of their transitions can be laid down under DST as presented in Table 1 . This is the case if the terminal perfectly follows logical operation flow and can be defined as deterministic terminal behaviour. In other words, this approach is based on estimating state duration and the moments of transition between states in line with terminal operation plan that does not include disturbances caused by unforeseen or hard to predict influences. The order of terminal operation flow in Table 1 under SST is derived considering the obtained state probabilities representing stochastic terminal behaviour. The moments of transition to the next state can be derived knowing the duration of each terminal state. Differences between deterministic and stochastic terminal behaviour in state transition order and state duration result in discrepancy between the moments when the terminal switches among the states.

After obtaining state probabilities for 12 steps in the SST method and deducing those in the DST method, a comparison of results with real-world operations flow for the year 2008 and the selection of the best-fit method for further short term planning follow. We evaluated the order of state transitions on a weekly basis and the fi-

Table 1. The duration of terminal states and the moments of transitions

\begin{tabular}{|c|c|c|c|c|c|c|c|c|c|c|c|c|c|c|}
\hline $\mathrm{DST}^{*}$ & $S_{1}$ & $S_{2}$ & $S_{3}$ & $S_{4}$ & $S_{5}$ & $S_{1}$ & $S_{2}$ & $S_{3}$ & $S_{4}$ & $S_{5}$ & $S_{1}$ & $S_{2}$ & $S_{3}$ & $\ldots$ \\
\hline Duration & 22 & 3 & 19 & 2 & 3 & 35 & 3 & 17 & 2 & 3 & 41 & 3 & 23 & \\
\hline $\begin{array}{l}\text { Mom. of } \\
\text { transition to } \\
\text { state }\end{array}$ & $0 \mathrm{~h}$ & $22 \mathrm{~h}$ & $\begin{array}{l}1 \mathrm{~d} \\
1 \mathrm{~h}\end{array}$ & $\begin{array}{c}1 \mathrm{~d} \\
20 \mathrm{~h}\end{array}$ & $\begin{array}{c}1 \mathrm{~d} \\
22 \mathrm{~h}\end{array}$ & $\begin{array}{l}2 \mathrm{~d} \\
1 \mathrm{~h}\end{array}$ & $\begin{array}{c}3 \mathrm{~d} \\
12 \mathrm{~h}\end{array}$ & $\begin{array}{c}3 \mathrm{~d} \\
15 \mathrm{~h}\end{array}$ & $\begin{array}{l}4 \mathrm{~d} \\
8 \mathrm{~h}\end{array}$ & $\begin{array}{c}4 \mathrm{~d} \\
10 \mathrm{~h}\end{array}$ & $\begin{array}{c}4 \mathrm{~d} \\
13 \mathrm{~h}\end{array}$ & $\begin{array}{l}6 \mathrm{~d} \\
6 \mathrm{~h}\end{array}$ & $\begin{array}{l}6 \mathrm{~d} \\
9 \mathrm{~h}\end{array}$ & $\begin{array}{l}7 \mathrm{~d} \\
8 \mathrm{~h}\end{array}$ \\
\hline $\mathrm{SST}^{*}$ & $S_{1}$ & $S_{2}$ & $S_{3}$ & $S_{4}$ & $S_{5}$ & $S_{2}$ & $S_{3}$ & $S_{3}$ & $S_{4}$ & $S_{1}$ & $S_{2}$ & $S_{3}$ & $S_{4}$ & $\ldots$ \\
\hline Duration & 23 & 3.5 & 20.5 & 2.5 & 4 & 4 & 19.5 & 11 & 1.5 & 43.5 & 3 & 25.5 & 2 & \\
\hline $\begin{array}{l}\text { Mom. of } \\
\text { transition to } \\
\text { state }\end{array}$ & $0 \mathrm{~h}$ & $23 \mathrm{~h}$ & $\begin{array}{c}1 \mathrm{~d} \\
2.5 \mathrm{~h}\end{array}$ & $\begin{array}{c}1 \mathrm{~d} \\
23 \mathrm{~h}\end{array}$ & $\begin{array}{c}2 \mathrm{~d} \\
1.5 \mathrm{~h}\end{array}$ & $\begin{array}{c}2 \mathrm{~d} \\
5.5 \mathrm{~h}\end{array}$ & $\begin{array}{c}2 \mathrm{~d} \\
9.5 \mathrm{~h}\end{array}$ & $\begin{array}{l}3 \mathrm{~d} \\
5 \mathrm{~h}\end{array}$ & $\begin{array}{c}3 \mathrm{~d} \\
16 \mathrm{~h}\end{array}$ & $\begin{array}{c}3 \mathrm{~d} \\
17.5 \mathrm{~h}\end{array}$ & $\begin{array}{c}5 \mathrm{~d} \\
13 \mathrm{~h}\end{array}$ & $\begin{array}{c}5 \mathrm{~d} \\
16 \mathrm{~h}\end{array}$ & $\begin{array}{c}6 \mathrm{~d} \\
17.5 \mathrm{~h}\end{array}$ & $\begin{array}{c}6 \mathrm{~d} \\
19.5 \mathrm{~h}\end{array}$ \\
\hline
\end{tabular}

* DST - represents a deterministic flow of terminal operations, SST - represents stochastic terminal operations 
nal results showed that the data obtained using the SST method matched practice in 38 cases (weeks), whereas applying the DST method embraced 9 cases and in 5 cases state transitions followed some other order. We may conclude that the observed terminal had stochastic behaviour in $73 \%$ cases in 2008 .

A comparison of the DST solution to the corresponding SST solution indicates that the later one better emulates the logic of bulk terminal operations flow. Therefore, if under the existing situation and without overtaking specific measures for improving operational effectiveness port management follows the SST method in planning short term operations in the terminal, the plan is expected to be more feasible. However, bearing in mind that SST terminal operations draw longer working procedures and therefore time lost on overcoming the effects of unforeseen events consume more resources, to perform even better, management should strive for adhering to the plan based on the DST method. Terminal operations that follow the DST method will certainly reduce operational costs as shown in the following section. For example, see Machuca et al. (2007).

\subsection{Cost Comparison}

Considering that limiting probability $\omega_{j}$ gives the long run mean fraction of time that the terminal is in state $j$ and each visit to state $j$ incurs a cost of $c_{j}$, then the long run mean cost per unit time $C$ is $C=\sum_{j=0}^{n} \omega_{j} c_{j}$.

The cost distribution of DST terminal operations obtained from Bakar terminal financial plan for each of the five states is $C_{D S T}=(1.00,2.37,5.21,1.93,3.05)$. The cost of each particular state represents the sum of operational costs providing that terminal operations follow deterministic states and transitions order without any stochastic influences, such as machinery failure, bad weather or the strike of longshoremen. The cost of the idle state has been taken as a referential and the cost of other states have been reduced to it. As a result, cost distribution and $\omega_{j}=0.2$ lead to the long run mean cost per unit time of 2.712 .

Limiting distribution for stochastic terminal operations is $\omega=(0.19,0.21,0.23,0.22,0.15)$. The cost distribution of SST terminal operations obtained from Bakar terminal financial datasheets for the year 2007 and for the cases when operations did not follow deterministic states and transitions order is $C_{S S T}=(1.00,2.57,5.63$, $2.09,3.28)$. Consequently, the long run mean cost per unit time equals to 2.972 . The cost distribution of SST terminal operations differs from that of DST which can be explained by various stochastic influences present in SST terminal operations. As expected, each unanticipated and therefore unplanned event draws extra costs in all states except the idle state. Difference in the long run mean cost per unit time equalling to $9.6 \%$ is considerable, although comprehensible SST terminal operations require additional manpower, equipment and overtime work. Also, it generates time wastage of waiting for work related decisions to be made.
The proposed procedures for reducing the effect of stochastic events include timely data collection on ship scheduling and expected time of arrival, hinterland connection congestions, cargo distribution and quantity and weather forecast. Besides, it is vital to keep regular maintenance, testing and control of equipment, education and training of employees as well as carrying out appropriate drills. The measures that are particularly effective comprise proper contingency planning and adhering to contingency procedures in case of the occurrence of an event that disturbs planned terminal operations. This way, if the terminal operates in line with a working plan, even if it is a contingency plan, the operations will be departing back from SST to DST mode. In order to make the contingency plans as appropriate as possible for real situations, it is important to having collected and analyzed data on past terminal operations under the influence of adverse events applying an appropriate method for creating practical contingency procedures.

\section{Conclusions}

1. We have answered the question whether the observed port terminal behaves as a deterministic system, i.e. according to the logical terminal operation flow, or as a stochastic system with a degree of variation in the order of terminal states.

2. The method may be used for short term tactical decision making identifying a particular state in which the terminal will be at a given instant. One of the major shortcomings of SST compared to DST terminal operations is represented by time lost and the consumption of more resources on overcoming the effects of unforeseen events resulting in the inefficiency of operational costs.

3. While the results are not as close to optimality as port management would desire, this is a tough reallife problem and an attempt to solve it is deriving high-quality solutions quickly which is essential for any practical application. The presented ST model can serve as a theoretical base for modelling the technological operations of other port terminals and traffic systems.

\section{References}

Afandizadeh, Sh.; Moayedfar, R. 2008. The feasibility study on creation of freight village in Hormozgan province, Transport 23(2): 167-171. doi:10.3846/1648-4142.2008.23.167-171

Česnauskis, M. 2007. Model for probabilistic assessment of oil outflow event caused by tanker accident, Transport 22(3): 187-194.

Chen, C.; Zeng, Q. 2010. Designing container shipping network under changing demand and freight rates, Transport 25(1): 46-57. doi:10.3846/transport.2010.07

Chang, Y.-L. 2003. WinQSB: Version 2.0. John Wiley \& Sons. $240 \mathrm{p}$.

Cullinane, K.; Song, D-W.; Wang, T. 2005. The application of mathematical programming approaches to estimating container port production efficiency, Journal of Productivity Analysis 24(1): 73-92. doi:10.1007/s11123-005-3041-9 
Cullinane, K. 2002. The Productivity and Efficiency of Ports and Terminals: Methods and Applications, in Grammenos, C. T. (Eds.) The Handbook of Maritime Economics and Business. London: LLP, 268-283.

Gendreau, M.; Laporte, G.; Séguin, R. 1996. Stochastic vehicle routing, European Journal of Operational Research 88(1): 3-12. doi:10.1016/0377-2217(95)00050-X

Hess, M.; Kos, S.; Hess, S. 2007. Queueing system in optimization function of port's bulk unloading terminal, $P R O-$ MET - Traffic \& Transportation 19(2): 61-70.

Hess, M.; Hess, S.; Kos, S. 2008. On transportation system with deterministic service time, PROMET - Traffic \& Transportation 20(5): 283-290.

Hsu, C.-I.; Hsieh, Y.-P. 2007. Routing, ship size, and sailing frequency decision-making for a maritime hub-and-spoke container network, Mathematical and Computer Modelling 45(7-8): 899-916. doi:10.1016/j.mcm.2006.08.012

Imai, A.; Shintani, K.; Papadimitriou, S. 2009. Multi-port vs. hub-and-spoke port calls by containerships, Transportation Research Part E: Logistics and Transportation Review 45(5): 740-757. doi:10.1016/j.tre.2009.01.002

Jaržemskis, A.; Vasilis Vasiliauskas, A. 2007. Research on dry port concept as intermodal node, Transport 22(3): 207-213.

Kia, M.; Shayan, E.; Ghotb, F. 2002. Investigation of port capacity under a new approach by computer simulation, Computer \& Industrial Engineering 42(2-4): 533-540. doi:10.1016/ S0360-8352(02)00051-7

Liu, W.; Xu, H.; Zhao, X. 2009. Agile service oriented shipping companies in the container terminal, Transport 24(2): 143-153. doi:10.3846/1648-4142.2009.24.143-153

Machuca, J. A. D.; Gonza'lez-Zamora, M. del M.; AguilarEscobar, V. G. 2007. Service operations management research, Journal of Operations Management 25(3): 585-603. doi:10.1016/j.jom.2006.04.005

Paulauskas, V.; Bentzen, K. 2008. Sea motorways as a part of the logistics chain, Transport 23(3): 202-207. doi:10.3846/1648-4142.2008.23.202-207

Radić, Z.; Bošnjak, I. 1997. Generalized traffic model and traffic equations derived from ST-diagrams, Modern Traffic 17(3/4): 237-241.

Su, T.-J.; Wang, P. 2009. Carrier's liability under international maritime conventions and the UNCITRAL draft convention on contracts for the international carriage of goods wholly or partly by sea, Transport 24(4): 345-351. doi:10.3846/1648-4142.2009.24.345-351

Vasilis Vasiliauskas, A.; Barysienè, J. 2008. An economic evaluation model of the logistic system based on container transportation, Transport 23(4): 311-315. doi:10.3846/1648-4142.2008.23.311-315

Veeke, H. P. M.; Ottjes, J. A. 1999. Detailed simulation of the containerflows for the IPSI-concept, in Simulation in Industry'99: 11th European Simulation Symposium, 220-223.

Wang, T.-F; Song, D.-W.; Cullinane, K. 2002. The applicability of data envelopment analysis to efficiency measurement of container ports, in Proceedings of the International Association of Maritime Economists (IAME) Conference. 13-15 November 2002, Panama, 95-111. 